\title{
Requirements, Methods and Research Issues for Modeling the Product Realization Process
}

\author{
K. W. Lyons
}

Manufacturing Systems Engineering Division

National Institute of Standards and Technology

Gaithersburg, MD, USA

301-975-6550, FAX 301-258-9749, klyons@cme.nist.gov

\author{
M. R. Duffey \\ Department of Engineering Management \\ George Washington University \\ Washington, D.C., USA \\ 202-994-7173, FAX 202-994-4606,duffey@seas.gwu.edu
}

\begin{abstract}
This paper presents a bottom up introduction to process modeling requirements for product realization which should drive new methods and computer tools. Though relatively high-level and organizational in scope, these models are attracting interest among design engineers, system engineers and technical managers whose decisions affect the complex downstream task interactions that cross organizational boundaries. A recent study of industrial usage, industrial requirements, and research issues is presented.
\end{abstract}

\section{Keywords}

Process Modeling, PERT, Product Realization Process, Enterprise Modeling, Business Process Re-engineering, BPR, IDEF, Petri Net, Project Management, PM

\section{OBJECTIVE}

Manufacturing firms in the U.S. and worldwide use various types of process models to study the business and engineering processes they use to "realize" their products, often in an effort to "Re-engineer the Enterprise". These process models are documented in a variety of forms and levels 
of detail yet the underlying objective is establish a strategy for assuring that the essential business and technical considerations related to a product's development are considered, evaluated, and understood by the total enterprise[1]. These models which typically represent the current ("as is") process can, in some cases, be used to explore and predict the impact of proposed ("to be") process changes. This paper explores how information and process models are being created and utilized by industry and attempts to identify key industry needs to assist in setting directions for future process modeling functionality extensions and standards efforts.

\section{STATEMENT OF PROBLEM}

Most process models in use by industry are relatively high-level and organizational in scope, yet their importance is just now being recognized by enterprises. Unfortunately, many companies fail to emphasize process modeling activities until the company is in a crisis thus looking for immediate solutions to problems that developed over extended periods. Enterprises most often use these methods when they require major restructuring to respond to current market place requirements yet they are also used by companies to support continuous quality improvement. An example of the latter might be in developing an approach to achieving improved concurrent product and process development which emphasizes process models for better coordination of design and manufacturing thus faster product development[2].

Although a significant effort is required to model processes, enterprises acknowledge its necessity. Identification and re-engineering of problem processes require as complete and unambiguous an understanding as possible of current product realization processes. For example, realization of electro-mechanical products involves complex task interactions across functional groups and departments which profoundly impact cost, quality, reliability and time-to-market. However, these interactions are extremely hard to visualize during early design stages. This raises a question: how might new computer-based tools enhance process modeling?

This report presents some key industry requirements and research issues for process modeling in design and manufacturing and identifies important functionality. Observations on the current state of process modeling and its limited acceptance by industry include the following:

- Process models are very tedious to develop and difficult to maintain currency. Most companies that have committed a major effort in this area are still using non-computer interpretable models (static) that are used to help "guide" new process development. This representation further compounds maintainability concerns.

-Process models are reasonably useful for visualizing process flow at multiple levels of abstraction. But beyond simply documenting activity precedence in an acyclic directed graph, process models have only very limited capabilities to characterize design iteration, allow simulation for schedule and cost, and represent time-dependent information flow in the enterprise.

- Process model accuracy and precision are inherently limited by subjective descriptions (in contrast with process planning paradigms in manufacturing) of human tasks and task interactions. There are also significant trade-off between clarity of the model (by limiting details of complex interactions) and the increased modeling effort required for precise output metrics.

- Process modeling techniques (current and emerging) require access to actual business and 
technical data from diverse functional groups within commercial manufacturing organizations. For many industrial firms, both the methodology and data in their process models have been understandably proprietary because of its competitive significance. Hence, there has been relatively little dissemination which inhibits meaningful research. Also, new mechanisms to validate advanced model concepts are required.

- Process models for electro-mechanical systems are inherently more difficult than for VLSI design, and probably even software design. Product complexity breeds process complexity, and the mechanical component interactions of geometry, heat, vibration, diverse fabrication constraints, etc. can make it difficult to predict process steps and sequences beforehand.

Despite these caveats, there is a resurgence of research efforts in developing new process modelers because of their potential pay-off for many different applications: documenting existing best practices, identifying bottlenecks (e.g., resource constraints) and task redundancy; "what if" analyses of design alternatives; risk assessment for schedule and cost; archiving processes; training; and many others.

\section{SOLUTION METHODOLOGY}

It is well known and quoted often that to "re-engineer a process" requires a thorough understanding of the existing process. To support this effort it is essential that adequate tools and methods are used to provide structure and a means to capture and document the process knowledge.

\subsection{Definition of Process Realization Process (PRP) modeling}

Although process modeling methods have been applied to many types of development efforts (e.g., software engineering, VLSI) our sole focus in this report is realization of discrete electro-mechanical products. The term PRP model (Product Realization Process model) will be used in this report to help reduce the ambiguity of the more generic term "process model", particularly as it relates to strictly physical processes in discrete and continuous flow manufacturing. Definitions of "process model" which have relevance include [3-5]. For purposes of discussion, the following working definition is presented:
A PRP model is a computer-interpretable description of the human and machine activities and their interactions required to realize a mechanical or electro-mechanical product. This may include early concept and configuration design activities, detailed design, prototyping, testing, tooling, fabrication, assembly and the many other activities within the scope of the realization process.

What it is ...

A PRP model should at least be a procedural model which documents precedence relationships between activities in a directed graph, and serve as a visual aid. A more robust PRP model is parametric, and its activity representations contain attribute/value pairs for assigned resources, duration times, cost rates, etc. By using stochastic values in a parametric PRP model, simulation techniques can generate estimates of total completion time, total cost, resource utilization, and other 
aggregate metrics for the entire process [6]. However, there are serious obstacles to valid parametric models given the complexity, partial definition, and uncertainty of real-world product realization efforts, which are discussed later in this report.

\section{What it is not ...}

- It is not an organization model. A process model's scope typically crosses departmental boundaries and in fact may point to mismatches between departmental responsibility and task requirements.

- It is not an information model. Its primary representation of process sequence and related time-dependencies distinguishes a process model from an information model.

- It is not just a flowchart of the generic sequence of design reviews and go/no-go decisions mandated within a particular corporate environment, without reference to a specific design and manufacturing effort.

It is not a discrete event simulator for machine-executable production processes.

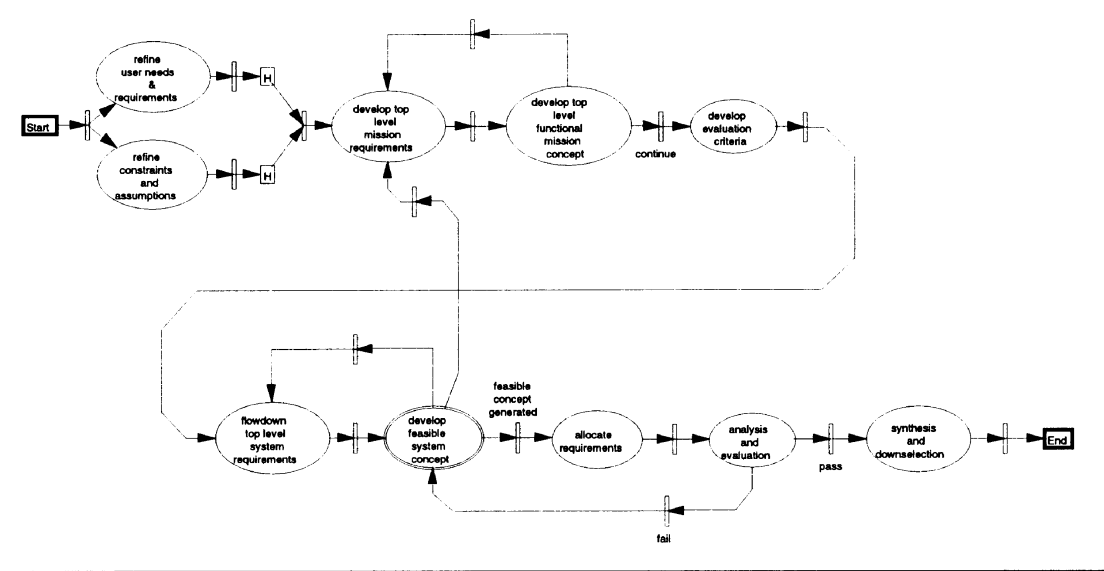

Figure 1: NASA satellite Pre-Conceptual Design PRP model using Modified Petri Net methodology (Part of an on-going collaborative effort with NASA GSFC)

\subsection{Modeling concepts and methodologies employed for study}

To assist in this study a tool that was recently developed and is currently being employed in several government research projects was used (Figure 1). The tool uses a Modified Petri Net (MPN) methodology and notation and has some advanced (yet largely untested) modeling features, such as representation of iteration cycles, conditional paths, shared resources and interdependencies between parallel or concurrent activities [7]. AN MPN graph offers a hierarchical decomposition similar to IDEF representations: each activity within an MPN can be made a "parent tasknet" which points to a subnet of sub-activities at a lower level of abstraction. Associated with each activity and event is a "frame" which can include information such as activity duration, resource requirements, knowledge-based rules, and conditional or probabilistic expressions for controlling activity 
parameters or process flow during simulation. A resource hierarchy is represented separately and can be linked to appropriate frames in the MPN. Various resource classes can be defined such as people, machines, and tools, along with multiple subclasses and, ultimately the resource instances that perform process activities. Once constructed, this MPN model can be executed via a built-in discrete event simulation routine that can be visualized as a process flow animation. Various activity states during simulation are monitored so that things such as resource utilization patterns, conflicts, and process bottlenecks can be identified.

\section{$4 \quad$ MAJOR RESULTS}

\subsection{Advanced PRP computer functionality and capabilities}

Companies that have made major commitments supporting continuous quality improvement usually have like efforts aimed at understanding their business and engineering processes. Such efforts, unfortunately, are still using non-computer-interpretable models to help "guide" new process development. These process development guidelines are very tedious and time consuming to develop and their static representation further compounds maintainability concerns (e.g., forces extensions and enhancements of the models to conform to scheduled update procedures). Companies are also looking at ways that the models can go beyond documentation of current ("as is") operations to supporting exploration of proposed ("to be") process changes and predict the result of these changes. To do this will require the development of new PRP applications and the extension of current PRP applications with advanced functionality.

What are the decision making needs of engineers and managers which should drive development of advanced PRP modeling tools? These tools should help evaluate the downstream implications of complex design process interactions that span traditional engineering departmental responsibilities. Some areas that are key to industrial acceptance of the tools are:

\section{Open Systems}

A major deficiency in currently available systems is that they are not "open." Very important product and process information remains locked up in the application and unavailable for use or access by others. This is very evident today as companies are increasingly concerned with capturing and retaining corporate knowledge to maintain their edge in a competitive marketplace. It is this corporate knowledge that is key to a company's success and typically not the applications and tools that the company uses. With the investment that companies are making to develop these models and the key information that they contain there needs to be some common representation or access (i.e., open architecture) so that companies have the capability to migrate or share information with other process modelers and engineering/manufacturing applications.

One approach to the sharing of information is conversion algorithms. Industry demand for conversion algorithms is expected to rise just as Computer Aided Design (CAD) file conversion routines have become a persistent (and still poorly met) demand of industry CAD users in the last fifteen years. Vendors of IDEF-based software have begun to provide output capabilities for both popular project management software and simulation software [7]. From the other end, several simulation software vendors are developing routines to input legacy IDEF models for their proprietary activity network representations. In these specific instances information or established links between information items can still be lost since most process models consist of both the 
formal model and supporting editorial and technical materials [8]. Beyond these vendor-specific efforts, there has evidently been little research in this area. Other information exchange mechanisms such as neutral files and open-architecture based applications can also assist industry in addressing this major acceptance and implementation barrier.

\section{Advanced Representations and Accuracy Issues}

Iterations: With all the uncertainties on designing products and the processes required to support the product, iterations remain as one of the most difficult topics to concisely represent in a model. Iterations are difficult events to predict and thus model, yet they are important components of engineering and manufacturing processes. While the general perception of iterative activities is negative there are times when it is desired. What is really required is a way to control iteration. To control iterations there needs to be recognition of when it occurs; both productive occurrences such as when it aids in the creative design process [9], and non-productive occurrences such as major redesign of products due to the late identification of assembly problems.

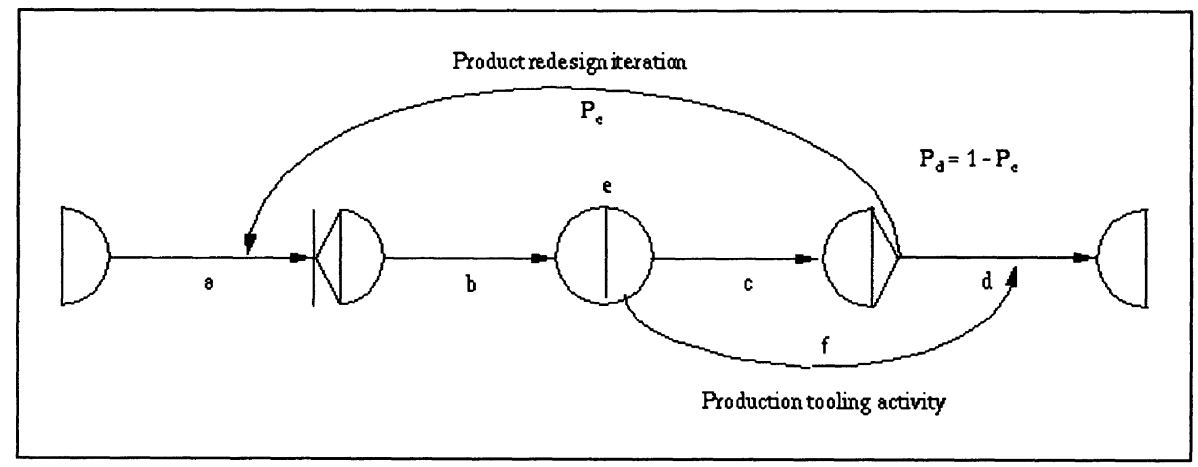

Figure 2: Example of a design iteration

Design iteration is a loosely defined term which describes the cycling of subgroups of activities typical in most PRP processes. Two types of activity interrelationships that are difficult or impossible to model with existing PRP methods are: i) iteration which occurs after concept reviews, prototype testing and other early phases of engineering design, and ii) changes to or cancellation of other concurrent manufacturing activities when redesign iterations occur. The use of iterative looping in a stochastic activity network is an imperfect yet perhaps potentially useful representation of the uncertainty associated with activity overlapping as described by [10] and identified by many companies as concurrent or simultaneous engineering of product and process. Consider modeling process flow in a simplified network for concurrent design and tooling in Figure 2 (an activity-on-arc representation described by Elmahraby [11]). When the redesign iteration occurs, the production tooling activity $f$ must terminate at the same point in system time when the iteration activity $e$ is realized (i.e., the duration for $f$ is not statistically independent). When activities $b, c$, and $f$ then occur for the second time, the parameters for the distribution functions of the duration times of $b, c$, and $f$ have changed. Similarly, the branching probability $p_{\mathrm{e}}$ is typically reduced after each successive redesign loop. 
Complexity: As product complexity increases a like increase in modeling complexity occurs. In addition, the complexity of the product to be designed affects the company's approach to defining the engineering and manufacturing processes to realize it. If product complexity and related issues can be modeled earlier in the design cycle at an adequate accuracy level it could significantly impact a company's success in the marketplace. One example might entail the use of a model to make key business decisions such as whether to make a specific product sub-system in-house or purchase from another company that has specific knowledge and core competency related to the sub-system. These make-buy decisions often are made with limited consideration of system level knowledge taking into account only component or sub-assembly attributes. Explicit representation of iterations based on historical patterns experienced by companies can capture complexity related systems problems.

Fidelity: A satisfactory model should contain the appropriate detail that is required to arrive at the correct decision. Too detailed a model requires substantial resource and time commitments that may not be reflected in savings to the company (ROI, Return on Investment). Knowing the right level of detail in creating the model depends on the experience of the person modeling the process.

Uncertainty: Using simulation to model uncertainty in a PRP has some unique problems. While manufacturing simulations typically model steady-state workflow conditions in a sequence of production operations, simulation of a product realization process must model a single unit, the design itself, as it increases in complexity of informational and physical detail by undergoing tasks within multiple organizational departments. Unlike in a manufacturing process, the description of the unit itself is not predefined, and is actually being transformed during its development. Because of this, there is uncertainty inherent in task duration and iteration that is not present in manufacturing simulation. Time variability in manufacturing simulations is typically aggregated from delay times for queuing, machine down-time, etc. and largely deterministic machine times in a production sequence. However, in a PRP simulation, virtually all task times have variability and iterative loops must be characterized. Nonetheless, PRP simulation merits research since traditional computerbased project management metrics for time, cost, resources, etc. are often inadequate.

Resources: To model a process the resources associated with the activities and events is key information. Many times comparison of "as is" and "to be" conditions pertain to the elimination, addition, or modification of certain resources (e.g., draft documents that are refined into final form). The resource can be a person, computer tool, documentation, or equipment. PRP systems need to be able to dynamically model resources usage and dependencies and minimize the need for apriori definition. In addition, the model should link the associated statistics that define the resource's availability and anticipated usage during the activity [12].

\section{Work Flow Management}

When a PRP model of sufficient detail and accuracy has been developed, and simulations run and validated, it can serve as a basis for exploring work flow management (WFM). This area needs further study to determine how specific issues unique to enactment of activities (e.g., generation of, and receipt of messages) and model complexity differ between simulation and enactment models [13]. Since simulation of engineering process models is based on a simplification/approximation 
of real processes there needs to be excellent correlation to what actually occurs under all conditions prior to its use in WFM as a controller of these engineering processes.

Initially it is expected that only portions of the PRP model will be used in the WFM environment where timing and coordination of activities are well defined. An example of WFM is the calling of design applications and automatic loading of specific models such as CAD 3-D models. Current application of WFM technologies have been most successfully applied in areas that have good structure and are deterministic (e.g., activity duration that are sequential with no iteration). Until accurate representations of processes are possible, WFM applications tend to be restrictive to users, and to address this condition most systems that are in production use today typically support a limited set of activities.

\subsection{Other issues relevant to PRP computer functionality and capabilities}

\section{Subjectivity}

Because PRP models represent sequences of human-executable as well as machine executable activities, the structure of a PRP model is inherently subjective in its capture of information flow. Modeling implications of subjectivity can be categorized into two areas: 1) the configuration of the model (e.g., defining activities and their precedence relationships in a directed graph), and 2) the content of the model (e.g., values of activity attributes which allow parametric characterization of activity duration, branching probabilities, and quantitative evaluation of time, cost, etc.). Beyond simply documenting activity precedence, how effectively can PRP models provide aggregate, quantitative metrics such as total cost and project completion time? This raises issues about the subjectivity of content in a process model such as estimation of activity duration time and assignment of branching probability values (for example, the pitfalls of assuming beta distributions for modeling time uncertainty in probabilistic PERT models).

\section{Information flow}

Unlike the clear sequence of fabrication activities in physical process planning, PRP models must often characterize a very ambiguous information flow. The natural tendency for those constructing the model is to define activities in terms of tangible inputs and outputs such as written specifications, analysis results, and material transformations. However, much information flow in a PRP is not easily specified, such as the informal communication network built up in an interdepartmental concurrent engineering team. Documenting these transactions is somewhat analogous to problems with the knowledge acquisition process acknowledged for expert systems development.

\section{Organizational issues}

The nature of organizational behavior makes a rigorous mathematical definition of its process semantics difficult. In addition, PRP models are very dependent on the imposed organizational constraints which will determine the optimal PRP considering company or industry specific constraints.

Cultural and institutional values: A company's or organization's culture, established principles and history often determines the approach and resulting "look" of the process models. Some organizations use specific groups skilled in enterprise modeling whereas other companies spread the task throughout the different organizations that comprise the enterprise. Company culture and 
institutional values can also determine the company's "inertia" which can prevent change from occurring, or at a minimum, slow it down.

Organization structure: Organizational structure can have significant effects on the development of a PRP model. Inherent within organizational structures are imposed constraints and limits that direct how process models will be developed. For organizations that are undergoing a major restructuring or re-engineering of the enterprise, the organizational aspects are many times less significant when it has been recognized that the current organizational structure is not effective. With smaller scoped re-engineering efforts this is usually not the case and models will tend to follow the along guidelines established to conform to organizational infra-structure. In many cases this organizational infrastructure is what provides the competitive edge for companies in responding to customer requests.

In some cases, a company's competitive environment can impair its ability to recognize or respond to change and make it vulnerable to new competitive attacks. An example of this is when a company competes against a select group of competitors and becomes complacent in this environment. The company knows what to expect and usually has sufficient knowledge to know when they are competitive. Their competitors often have very similar organizational structures and this structure in a static marketplace enables them to compete effectively as long as the marketplace can support them. Yet, when a new competitor enters the marketplace this company realizes that to be competitive they must approach the product development process differently. This change in the way "the game is played" can have severe consequences on the established companies if they fail to recognize this change and take actions to predict its impact on them. This example demonstrates where imposed organization structure can actually impede the recognition of new methods and approaches in developing the product and can have major repercussions.

Model acceptance: Modeling engineering processes is a very tedious and difficult activity and most often is done by individuals skilled primarily in process modeling, not typically in the engineering or manufacturing process being modeled. This approach has been traditionally viewed as the best practice since modeling required very specific skills to successfully interview engineering personnel and obtain the process knowledge and information in a form conducive to the modeling methodology. This was effective in that this skilled individual could rapidly construct a model that, from one view point, captured and accurately represented the process. Unfortunately, this approach can lead to models that fail to adequately represent the process in a way that is of value to the enterprise. Three reasons for these model inadequacies are:

- Lack of domain knowledge by the expert process modeler which prevents the modeler from being able to realize the inaccuracies of the model,

- Lack of commitment of engineering personnel ("buy-in") to validate the models and apathy toward the real application or need for the models, and

- Limitations of the modeling methods and tools which prevent adequate representation of key process attributes.

To initiate a modeling effort it can be useful to start with existing process information for assisting in model creation. This can help achieve acceptance by jump starting the modeling effect yet can also have some unanticipated and potentially significant effects. Many times the most useful documents are PERT charts used for project management (PM). Although these materials help reduce the time in generating a model there is some concern that it can bias a PRP model by filtering the intended enterprise view with a project management perspective. It is difficult to surmise the 
impact of biasing PRP models with PM information. Depending on how the PRP models are to be used and what results are required will dictate what information should be used for model creation. Awareness of the potential for biasing the model is the first step in minimizing this effect.

\subsection{Future Directions}

Many of these have been touched on in previous sections. Others include:

- Standards-related research

- Methods to assign different levels of confidence, different representational constructs, with different levels of abstraction

- Identifying feedback loops and iteration

- Multi-media process models

- Multiple viewpoints of a process an aid to DFX evaluations.

- Relationship of process model to both product representation (CAD models) and external data of the firm (e.g., financial data in accounting software)

- Explicit methodology for fine-tuning cost and time estimates as the project progresses.

- Application of advanced statistical techniques to eliminate distribution dependencies and improve predictions of complex series of activities.

\section{SUMMARY}

Product realization for electro-mechanical assemblies is a very complex, interdisciplinary process. It requires decision-making not only about attributes of the design itself, but also about resource allocation and scheduling for many product and manufacturing engineering activities, as well as extensive coordination with concomitant activities in marketing, finance, purchasing, and other functional groups inside and outside a company structure. Typically, complex interdependencies exist among these disparate activities, and it is difficult to predict how decisions will affect overall organizational objectives of low cost, high quality and short time-to-market.

Unfortunately, progress towards a comprehensive model of the product realization process falls in a gap between established research disciplines and industry practice. The value of modeling processes is still not realized, in that most companies still under utilize the information and knowledge contained in the models. Causes of this are found in the enterprise's approach to process modeling as well as the inefficiencies of currently available tools.

To gain better insight into these problems, case studies are critical in that they are based on actual industry experiences with supporting justification on the benefits that can result. Engineering designers have developed some very good models that guide decisions about product attributes as they affect cost and/or quality for an individual manufacturing activity. However, these models generally neglect interdependencies between activities and organizational constraints. At the other extreme, PRP managers have generally modeled the product development process as a comprehensive but highly abstract network of activities (such as PERT networks). These network models ideally assist decision-making about activity scheduling and resource allocation by providing very rough estimates of cost and time-to-market. However, the lack of detail and other limitations in such models obscure many important issues, such as the effects of activity interdependencies and sensitivity to changes in product attributes. 


\section{REFERENCES}

[1] National Research Council, Improving Engineering Design: Designing for Competitive Advantage, National Academy Press, Washington, D. C., 1991.

[2] Dertouzos, M., Lester, R., and Solow, R., Made in America: Regaining the Productive Edge, The MIT Press, Cambridge, Massachusetts, 1989.

[3] Busby, J.S. and Williams, G.M. "The value and limitations of using process models to describe the manufacturing organization." International Journal of Production Research, vol. 31, p.39, 1993.

[4] Curtis, Bill, Kellner, Marc, and Over, Kim. "Process Modeling", Communications of the ACM, vol.35, no.9, September 1992.

[5] Malone, Thomas W., Crowston, K., Lee, J. and Pentland, B. "Tools for Inventing Organizations: Toward a Handbook of Organizational Processes, "Proceedings of the 2nd IEEE Workshop on Enabling Technologies Infrastructure for Collaborative Enterprises, Morgantown, WV, April 2022, 1993.

[6] Duffey, Michael R., and Dixon, J.R. "Managing the Product Realization Process: A Model for Aggregate Cost and Time-to-Market Evaluation, "Journal of Concurrent Engineering: $\quad$ Research and Applications, no.1, vol.1, 1993.

[7] Madni, Azad M. "Computer-Aided Concurrent Engineering/Process Modeler: User's Manual,"Perceptronics, Inc., Woodland Hills, CA., 1994.

[8] Design/IDEF/ServiceModel: The relationships between Workflow Analysis Models and ServiceModel/ProModel. Meta Software Corporation, Cambridge, MA, February 1995.

[9] Schenck, D., and Wilson, P., Information Modeling: The EXPRESS Way, Oxford University Press, Oxford, U.K., 1994.

[10] Clausing, D., Total Quality Development: A Step-By-Step Guide to World-Class Concurrent Engineering, ASME Press, New York, NY, 1994.

[11] Elmaghraby, S.E., Activity Networks: Project Planning and Control by Network Models. John Wiley \& Sons, New York, 1977.

[12] Clark, Kim, and Fujimoto, Takahiro. "Overlapping Problem-Solving in Product Development," Managing International Manufacturing. Elsevier Science Publishers, New York, 1989. 
[13] Bonney, M., Barson, R., Head, M., and Huang, N., UNISON - A Tool for Enterprise Integration, in Enterprise Integration Modeling: Proceedings of the First International Conference (ed. C.J. Petri), MIT Press, Cambridge, Massachusetts, 1992.

\section{BIOGRAPHY}

Kevin W. Lyons - Mr. Lyons' current assignment involves design methodology, modeling methodologies for product realization processes, and design system integration in the domain of mechanical and electro-mechanical components, assemblies, and systems. He is also working with the ARPA MADE (Manufacturing Automation and Design Engineering) Program which involves coordination activities and applied research in design knowledge capture.

Mr. Lyons came to NIST with 15 years of industry experience with IBM. In IBM's Lexington, KY manufacturing facility. He had assignments in Product Assurance, Quality Engineering (Electrical and Mechanical), Development Engineering, and Computer-Aided-Design and Analysis Support, with key focus on assemblies.

Michael R. Duffey - Dr. Duffey is an Assistant Professor at George Washington University (GWU), Department of Engineering Management. Dr. Duffey joined the faculty at GWU in September 1992 after completing his Ph.D. in Mechanical Engineering at the University of Massachusetts (Amherst).

Dr. Duffey's current research interests include modeling methodologies for the product realization process, engineering economics, and curriculum development for adult technical training. He works closely with the National Institute of Standards and Technology (NIST) Engineering Design Technologies Group where he does collaborative research as a faculty researcher. 